\title{
DESPECKLING POLSAR IMAGES BASED ON RELATIVE TOTAL VARIATION MODEL
}

\author{
C. Jiang ${ }^{1,2, *}$, X.F. He $^{2}$, L.J. Yang ${ }^{1}$, J. Jiang ${ }^{1}$, D.Y. Wang ${ }^{1}$, Y. Yuan ${ }^{1}$ \\ ${ }^{1}$ Dept. of Suveying and Geoinformatics, Nanjing University of Posts and Telecommunications, Nanjing, P.R.China - (jiangc, yanglj, \\ jiangj,yuany)jiangc@njupt.edu.cn \\ ${ }^{2}$ School of Earth Sciences and Engineering, Hohai University, Nanjing, P.R.China - (hexf, jiangc)
}

\section{Commission III, WG III/2}

KEY WORDS: PolSAR, Despeckling, Relative Total Variationl, Wishart Measure, PolRTV

\begin{abstract}
:
Relatively total variation (RTV) algorithm, which can effectively decompose structure information and texture in image, is employed in extracting main structures of the image. However, applying the RTV directly to polarimetric SAR (PolSAR) image filtering will not preserve polarimetric information. A new RTV approach based on the complex Wishart distribution is proposed considering the polarimetric properties of PolSAR. The proposed polarization RTV (PolRTV) algorithm can be used for PolSAR image filtering. The L-band Airborne SAR (AIRSAR) San Francisco data is used to demonstrate the effectiveness of the proposed algorithm in speckle suppression, structural information preservation, and polarimetric property preservation.
\end{abstract}

\section{INTRODUCTION}

Polarimetric Synthetic Aperture Radar (PolSAR) is a new type of imaging radar used to measure the polarization characteristics of radiation signals. Because of its ability to obtain target scattering echoes in different polarimetric states, PolSAR is better to describe the information contained in the target and has been widely used in many fields including military investigation, geological exploration, surveying agriculture and forestry research. However, the speckle existing in PolSAR seriously hinders the quality of image and affects the classification, identification and interpretation. Therefore, the study of PolSAR speckle suppression algorithm has become an important problem in the application of PolSAR.

Different methods have proposed to reduce speckles on PolSAR, including adaptive filters, nonlocal(NL) means filters and variational methods. Adaptive filters are built based on the theory of minimum mean square error(MMSE), such as Lee filter, Kuan filter, Frost filter. Generally, these filters reduce the image speckle with local statistics and adjusting filter window. The NL means filters can well preserve the repetitive structures. However, these methods present huge computational burden.

In recent years, variational methods have been widely studied for SAR image despeckling since they can effectively reduce speckle preserving linear structure. The total variation(TV) despeckling model was proposed by Rudin, Osher, Fatemi(ROF) (Rudin et al.,1992). Since SAR images contains multiplicative speckle noise, Wang, et al. proposed to first transform the multiplicative noise into additive noise through logarithmic transformation and then applied TV despeckling method (Wang et al., 2017). To reduce the staircase effect, Markarian et al. proposed a method using high-order total variation (High-TV) despeckling, which is in the compressive sensing (CS) framework (Markarian et al., 2017). On PolSAR images, speckle perturbs each polarization channel and the interchannel correlations, which carry the important information on the target scattering mechanisms. Liu et al. proposed two adaptive vectorial models for despeckling multi-channel SAR images (Liu et al.,2013). However, the filtering methods process the channels respectively are not suitable for PolSAR data due to the complete target statistics are contained in the covariance matrix or coherent matrix of the multivariate scattering vector.

The purpose of this paper is to address the problem of PolSAR despeckling. In this paper, we propose a polarization relative TV (PolRTV) despeckling method for PolSAR image. The rest of this paper is organized as follows. In section 2, the PolRTV models for speckle reduction of PolSAR coherency is presented. Experimental results on real PolSAR data are shown in Section 3. Some concluding marks are given in Section 4.

\section{IMPROVED RELATIVE TOTAL VARIATION MODEL}

\subsection{Relative Work}

$\mathrm{Xu}$ et al. proposed the RTV model which make main structures stand out (Xu et al.,2012).The objective function is expressed as

$$
\arg \min _{S} \sum_{p}\left(S_{p}-I_{P}\right)^{2}+\lambda\left(\frac{D_{x}(p)}{L_{x}(p)+\varepsilon}+\frac{D_{y}(p)}{L_{y}(p)+\varepsilon}\right)
$$

where $\quad p=$ the index of $2 \mathrm{D}$ pixels

$I, S=$ the input image and extracted structures

$D_{x}(p), D_{y}(p)=$ windowed total variations in the $x$ and $y$ directions

$L_{x}(p), L_{y}(p)=$ windowed herent variations in the $x$ and $y$ directions 


$$
\begin{aligned}
& \lambda=\text { the weight in regularized optimization } \\
& \varepsilon=\text { the small positive number }
\end{aligned}
$$

\subsection{Model Formulation}

The PolSAR data involve complex-valued matrics with multiplicative and additive noise. The diagonal elements of the coherency matrix are real numbers and can be characerized by the multiplicative noise, while the off-diagonal elements are complex numbers and characterized by a combination of multiplicative and additive noise (Lopez-Martinez et al.,2003). To explore the mixed noise intensively from PolSAR, we aimed to formulate a new model from two aspects: (1) we wanted to apply a better way to capture the structure based on the diagonal elements of the coherency matrix; (2) we wanted to apply a better way to preserve the polarimetric information based on the off-diagonal elements of the coherency matrix

Considering the PolSAR data in the area of size $m \times n$ and every resolution element corresponds to a $3 \times 3$ coherency matrix, the objected coherency data $\mathbf{Z}$ and the true coherecy data $\mathbf{T}$ in this area are fourth-order tensors with size $m \times n \times 3 \times 3$. For convenience, we use the following notations:

$$
\begin{aligned}
& I=\{i \mid 1<\mathrm{i}<\mathrm{m}\}, J=\{j \mid 1<\mathrm{i}<\mathrm{n}\} \\
& L=\{l \mid 1<1<3\}, K=\{k \mid 1<\mathrm{k}<3\} \\
& T_{l, k}:=\mathbf{T}(:,:, l, k), Z_{l k}:=\mathbf{Z}(:,:, l, k) \\
& T^{d}:=\mathbf{T}(:,:, l, l), Z^{d}:=\mathbf{Z}(:,:, l, l) \\
& T^{\text {od }}:=\mathbf{T}(:,:, l, k), Z^{\text {od }}:=\mathbf{Z}(:,,:, l, k)
\end{aligned}
$$

where $d$ denotes the diagonal elements of coherency matrix, $d$ denotes the off-diagonal elements of coherency matrix, $T^{d}$ and $Z^{d}$ are $m \times n \times 3$ matrices, and $T^{o d}$ and $Z^{\text {od }}$ are $m \times n \times 6$ matrices.

In this paper, $\|\mathbf{T}\|_{T V}$ is the total difference between the adjacent coherency matrics. The discrete version of $\|\mathbf{T}\|_{T V}$ on the complex field as follows:

$$
\begin{aligned}
& \|\mathbf{T}\|_{T V}:=\sum_{i \in I} \sqrt{\sum_{j \in J}\left\|\nabla T_{i, j}\right\|_{F}^{2}} \\
& \left\|\nabla T_{i, j}\right\|_{F}^{2}=T_{(i, j)_{x}}^{*} T_{(i, j)_{x}}+T_{(i, j)_{y}}^{*} T_{(i, j)_{y}}
\end{aligned}
$$

where $\quad \nabla=$ the gradient operator

$$
\begin{aligned}
T_{(i, j)_{y}}, T_{(i, j)_{x}}= & \text { the first-order finite difference of the } \\
& \text { matrix }
\end{aligned}
$$

To preserve the structure and polarimetric infomation of PolSAR, we improved the RTV model as follows:

$$
\arg \min _{T}\left\{\sum_{i \in I, j \in J}\left[\operatorname{Tr}\left(T_{i, j}^{-1} Z_{i, j}\right)+\log \left(\operatorname{det}\left(T_{i, j}\right)\right)\right]+\tau\left(\frac{T^{d}(p)}{T^{d}(p)+\varepsilon}\right)+\lambda\|T\|_{T V}\right\}
$$

where

$$
\begin{aligned}
& \operatorname{Tr}(\cdot)=\text { the trace of matrix. } \\
& \operatorname{det}(\cdot)=\text { the determinant of matrix } \\
& \tau=\text { the weight of structure feature } \\
& \lambda=\text { the weight of polarimetric information }
\end{aligned}
$$

The diagonal elements of the polarized coherent matrix participate in the RTV. In iterative process, key steps of the iteration mainly depend on the wishart distance comparison between the two adjacent iteration results.

\section{EXPERIMENT AND RESULTS}

\subsection{Experimental Datasets}

The PolSAR datatset is acquired by the AIRSAR sytem over San Francisco. The region of $300 \times 300$ pixels is selected from the AIRSAR image, which is mainly composed of water, forest and urban areas. The Pauli decomposition image of the AIRSAR data is shown in Fig.1.

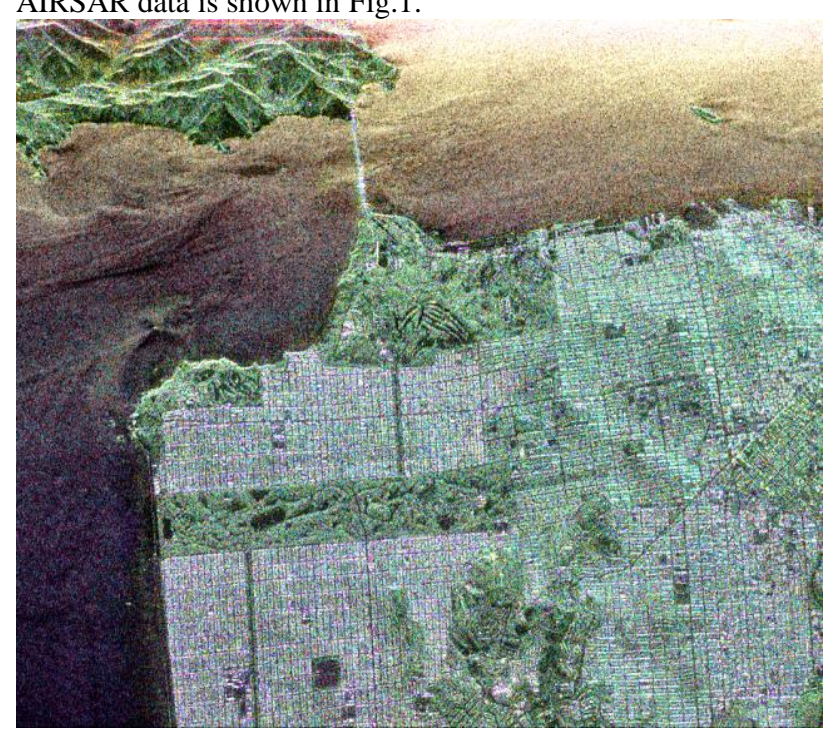

Figure 1. AIRSAR color-coded Pauli image of San Francisco.

\subsection{Evaluation}

For quantitative comparison, the equivalent number of looks (ENL), the structural similarity (SSIM), the polarimetric signature and Edge preservation degree based on ratio of average (EPD-ROA) are utilized in this paper.

3.2.1 Equivalent Number of Looks: The ENL is an indicator of speckle nosie level. Higher ENL value means perfect despeckle result (Huang et al.,2009), which is defined as follows:

$$
E N L=\frac{\mu^{2}}{\delta^{2}}
$$

where $\mu=$ the average intensity of the homogeneous area $\delta=$ the corresponding standard deviation.

3.2.2 Structural Similarity Index Measure: Wang et al. proposed SSIM to measure the similarity between two images (Wang et al., 2004). The values of the SSIM is in $[-1,1]$. SSIM is defined as follows:

$$
\begin{aligned}
& \operatorname{SSIM}(x, y)=\frac{\left(2 \mu_{x} \mu_{y}+C_{1}\right)\left(2 \sigma_{x y}+C_{2}\right)}{\left(\mu_{x}^{2}+\mu_{y}^{2}+C_{1}\right)\left(\sigma_{x}^{2}+\sigma_{y}^{2}+C_{2}\right)} ; M \operatorname{SSIM}=\frac{1}{p} \sum_{j=1}^{p} \operatorname{SSIM}_{j} \\
& \text { where } C_{1}, C_{2}, C_{3}=\text { small constants } \\
& \mu_{x}, \mu_{y}=\text { the mean of } \mathbf{x} \text { and } \mathbf{y} \text { respectively } \\
& \sigma_{x}, \sigma_{y}=\text { the variance of } \mathbf{x} \text { and } \mathbf{y} \text { respectively } \\
& \sigma_{x y}=\text { the variance of } \mathbf{x} \text { and } \mathbf{y}
\end{aligned}
$$


3.2.3 Polarimetric Copol and Crosspol Signatures: The polarimetric signature describes the scattering coefficient and allows measure the variation of the scattering coefficient with polarization, which is sensitive to different targets. The polarimetric signature $\sigma^{0}$ is reprented by the following equation:

$$
\sigma^{0}=K\left[\begin{array}{c}
1 \\
\cos \left(2 \phi_{i}\right) \cos \left(2 \psi_{i}\right) \\
\cos \left(2 \phi_{i}\right) \sin \left(2 \psi_{i}\right) \\
\sin \left(2 \phi_{i}\right)
\end{array}\right]^{T}\left[\begin{array}{c}
1 \\
\cos \left(2 \phi_{j}\right) \cos \left(2 \psi_{j}\right) \\
\cos \left(2 \phi_{j}\right) \sin \left(2 \psi_{j}\right) \\
\sin \left(2 \phi_{j}\right)
\end{array}\right]
$$

where

$$
\begin{aligned}
K= & \text { constants } \\
\phi_{i}, \psi_{i}= & \text { the ellipticity and ellipse orientation angles } \\
& \text { of the polarization of the receiving antenna } \\
\phi_{j}, \psi_{j}= & \text { the ellipticity and ellipse orientation angles } \\
& \text { of the polarization of the transmitting } \\
& \text { antenna }
\end{aligned}
$$

Both parameters $\phi$ and $\psi$ set to values usually respectively from $-45^{\circ}$ to $+45^{\circ}$ and from $-90^{\circ}$ to $+90^{\circ}$.

3.2.4 Edge Preservation Degree Based on Ratio of Average: The EPD-ROA indicates the ability of preserving the horizontal and vertical edge of the image. When the EDPROA is closer to one, it means preserving edge better. The EDP-ROA is between the noisy image $Y_{O}$ and the filtered image $\hat{X}_{D}$.

$$
E P I=\frac{\sum_{i=1}^{m}\left|\hat{X}_{D 1}(i) / \hat{X}_{D 2}(i)\right|}{\sum_{i=1}^{m}\left|Y_{O 1}(i) / Y_{O 2}(i)\right|}
$$

where $\hat{X}_{D 1}, \hat{X}_{D 2}=$ Two adjacent pixel values of the despeckled image along a certain direction(vertical (V) or horizontal $(\mathrm{H}))$

$Y_{O 1}, Y_{O 2}=$ Two adjacent pixel values of the speckled image along a certain direction(vertical (V) or horizontal $(\mathrm{H}))$

\subsection{Experiments on PolSAR Data}

The filtering experiments are performed and compared by the TV and non-TV filtering methods, respectively. In this paper, the chosen filtering methods includes (a) the Boxcar filter;(b) the intensity driven adaptive neighborhood (IDAN); (c) the Refined Lee filter; and (d) the proposed PolRTV filter.

In this experiments, the despeckled images are represented by calculating the ENL, the SSIM and the EPD-ROA for the whole image along the horizontal (EPRH) and vertical (EPRV) direction. Table 1 gives all the numerical evaluation results for these images. The despeckled results for the different filters are shown in Fig.2. We used the polarization signature to evaluate the preservation of polarimetric information for the filters. Figure 3 shows the copular and cross-polar signature for builtup arear.

As shown in Tab.1, these results are clearly in the indicate that the PolRTV method produces better results. Table 1 shows that the Refined Lee filter obtained the highest EPRV value in the vertical direction and the IDAN obtained the highest ENL value. Compared the other three filters, PolRTV obtains the highest SSIM value and EPRH value, which means the filter has the ability of preserving the structural information of image.

\begin{tabular}{|l|c|c|c|c|}
\hline Filter & ENL & SSIM & EPRH & EPRV \\
\hline Boxcar & 46.3952 & 0.1861 & 0.6981 & 0.7335 \\
IDAN & $\mathbf{5 3 . 7 2 7 2}$ & 0.1519 & 0.6980 & 0.7363 \\
Refined Lee & 25.9164. & $\mathbf{0 . 8 9 7 5}$ & $\mathbf{0 . 6 9 9 0}$ & $\mathbf{0 . 7 3 6 7}$ \\
PolRTV & $\mathbf{4 6 . 4 3 5 4}$ & $\mathbf{0 . 9 4 5 2}$ & $\mathbf{0 . 7 0 1 2}$ & $\mathbf{0 . 7 2 8 8}$ \\
\hline
\end{tabular}

Table 1. Quantitative evaluation of the filtering performance

Figure 2 shows the despeckling results in Pauli decompostion image. They indicate that the classical methods remove speckle noise in homogeneous area with fewer texture features to reflect in the heterogeneous area.

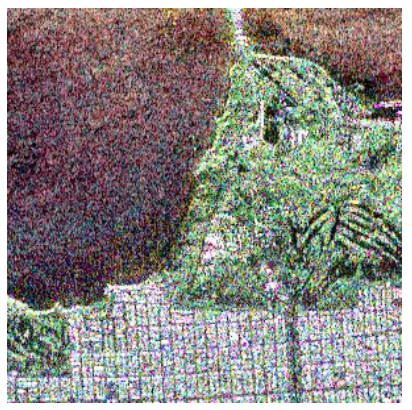

(a)

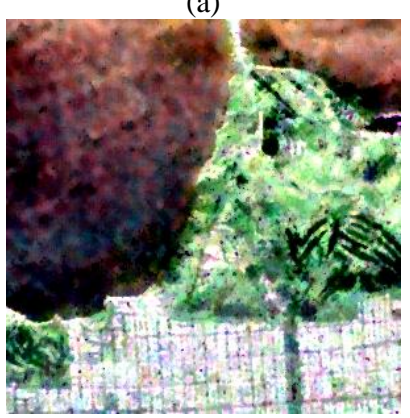

(c)

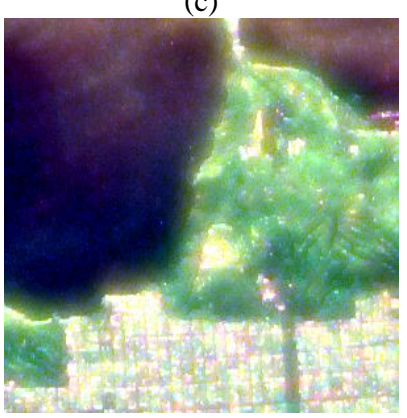

(e)

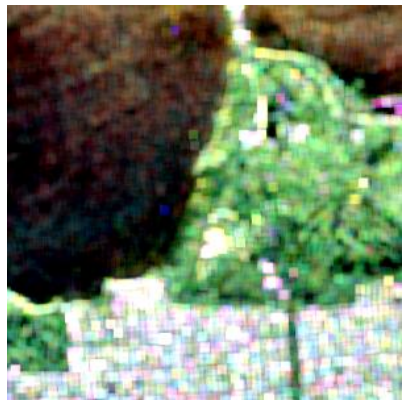

(b)

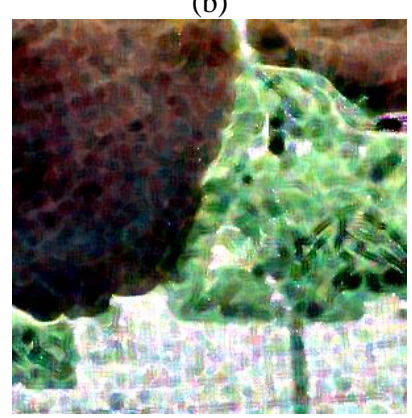

(d)
Figure 3. Pauli decomposition of the original AIRSAR image over San Francisco and its filtered results. (a) AIRSAR L-band data. (b) Boxcar filter. (c) IDAN filter. (d) Refined Lee filter. (e) PoRTV filter.

Polarimetric signatures are often used to visually evaluate the filtered results. In Fig. 3, we compare the five polarization response at the urban area. It is clear from these figures that the calculated polarization signatures are more or less different from each other. Compared with other filtered images, there are relative similarities between the polarization response provided 
by PolRTV filtered image and that of original image. Their copolarization and cross-polarization signatures show some peaks corresponding to the maximum backscattered power. Therefore, this result confirms that the polarimetric properties are well preserved by PolRTV filter.

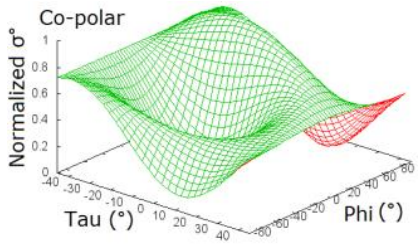

(a)

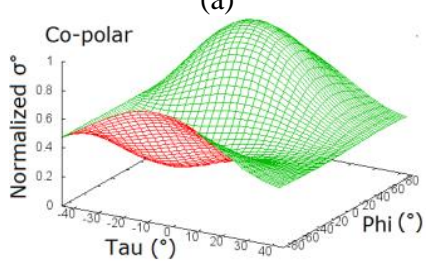

(c)

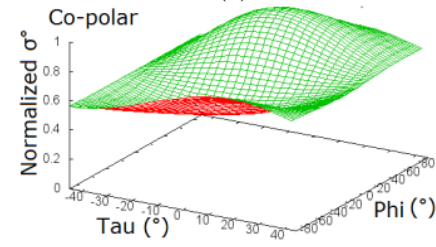

(e)

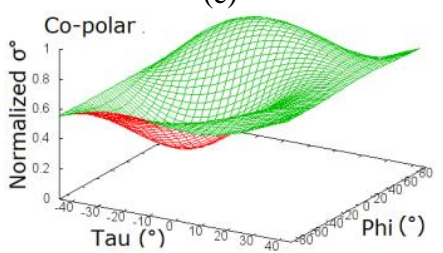

(g)

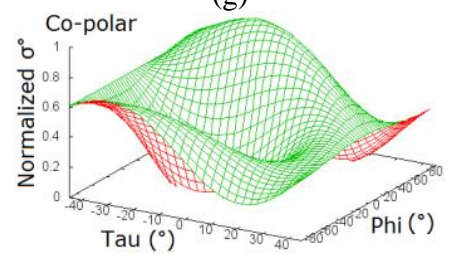

(i)

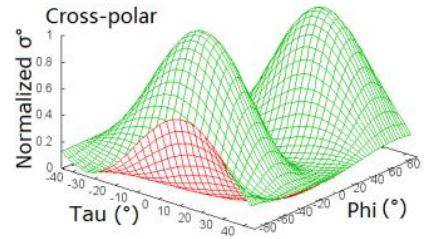

(b)

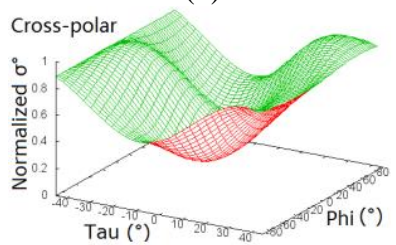

(d)

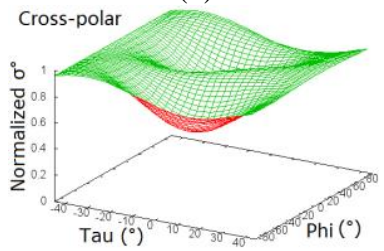

(f)

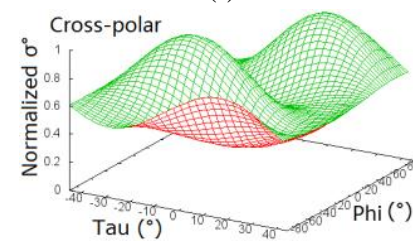

(h)

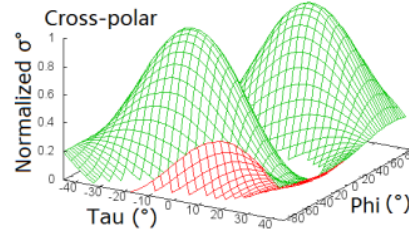

(j)
Figure 3. Co-polar and cross-polar polarization signature.(a,b) The original image.(c,d) Boxcar.(e,f) IDAN.(g,h) RefinedLee. (i,j) PolRTV

\section{CONCLUSION}

In this paper, we proposed a PolRTV despeckling method considering the wishart measure. The method preserves not only the structural information but also polarimetric properties of PolSAR image. However, in some extent, the method blurs the edge of region, such as coastline. Improving the method to preserve the edge of region and extending the method to extraction of different objects from PolSAR images are future works in this direction.

\section{ACKNOWLEDGEMENTS}

This study was supported by the National Science Foundation of China project (No. 41601497, No.61502246), the Natural
Science Foundation of Jiangsu Province (No. BK20140868, BK20170897) and the Nanjing University of Posts and Telecommunications (NUPTSF) (No. NY215181). The authors wish to thank Dr. Wenmei Li from NJUPT for her help in processing of PolSAR image. The authors would like to thank the European Space Agency for making available the AISSAR L-band data of San Francisco Bay. Also, the authors greatly appreciate the thoughtful comments and reviews of anonymous reviewers that helped to enhance this paper.

\section{REFERENCES}

Rudin, L. I., Osher, S., and Fatemi, E.,1992. Nonlinear total variation based noise removal algorithms. Physica D: Nonlinear Phenomena,60(1-4), pp. 259-268.

Wang, X., Jia, Z., Yang, J., and Kasabov, N., 2017. Change detection in sar images based on the logarithmic transformation and total variation denoising method. Remote Sensing Letters, 8(3), pp. 214-223.

Markarian, H., Ghofrani, S. 2017. High-TV based CS framework using map estimator for sar image enhancement. IEEE Journal of Selected Topics in Applied Earth Observations \& Remote Sensing, 10(9), pp. 1-15.

Liu, H., Yan, F., Zhu, J., and Fang, F., 2013. Adaptive vectorial total variation models for multi-channel synthetic aperture radar images despeckling with fast algorithms. IET Image Processing, 7(9), pp. 795-804.

Lopez-Martinez, C., and Fabregas, X., 2003. Polarimetric SAR speckle noise model. IEEE Transactions on Geoscience and Remote Sensing, 41(10), pp. 2232-2242.

Xu, L., Yan, Q., Xia, Y., and Jia, J.,2012. Structure extraction from texture via relative total variation. ACM Transactions on Graphics, 31(6), 139:1-139:10

Huang, S. Q., Liu, D. Z., Gao, G. Q., and Guo, X. J. 2009. A novel method for speckle noise reduction and ship target detection in SAR images. Journal of Pattern Recognition, 42(7), pp.1533-1542.

Wang, Z., Bovik, A. C., and Sheikh, H. R., and Simoncelli, E. P., 2004. Image quality assessment: from error visibility to structural similarity. IEEE Transactions on Image Processing, 13(4), pp. 600-612.

Feng, H., Hou, B., and Gong, M., 2014. SAR Image Despeckling based on local homogeneous-region segmentation by using pixel-relativity measurement. IEEE Transactions on Geoscience and Remote Sensing, 49(7), pp. 2724-2737. 